

University of Central Florida

STARS

$1-1-2014$

\title{
Utilization of Microscale Silicon Cantilevers to Assess Cellular Contractile Function In Vitro
}

\author{
Alec S. T. Smith \\ University of Central Florida \\ Christopher J. Long \\ University of Central Florida \\ Christopher McAleer \\ University of Central Florida \\ Nathaniel Bobbitt \\ University of Central Florida \\ Balaji Srinivasan \\ University of Central Florida \\ Find similar works at: https://stars.library.ucf.edu/facultybib2010 \\ University of Central Florida Libraries http://library.ucf.edu \\ See next page for additional authors \\ This Article is brought to you for free and open access by the Faculty Bibliography at STARS. It has been accepted for \\ inclusion in Faculty Bibliography 2010 s by an authorized administrator of STARS. For more information, please \\ contactSTARS@ucf.edu.
}

\section{Recommended Citation}

Smith, Alec S. T.; Long, Christopher J.; McAleer, Christopher; Bobbitt, Nathaniel; Srinivasan, Balaji; and Hickman, James J., "Utilization of Microscale Silicon Cantilevers to Assess Cellular Contractile Function In Vitro" (2014). Faculty Bibliography 2010s. 6097.

https://stars.library.ucf.edu/facultybib2010/6097

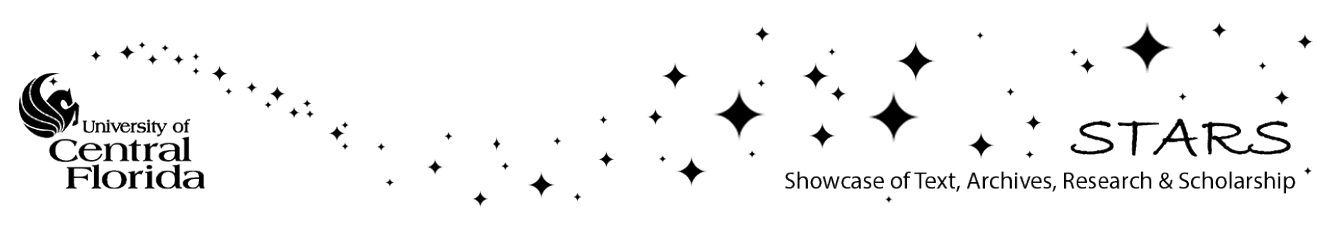




\section{Authors}

Alec S. T. Smith, Christopher J. Long, Christopher McAleer, Nathaniel Bobbitt, Balaji Srinivasan, and James J. Hickman 


\title{
Video Article \\ Utilization of Microscale Silicon Cantilevers to Assess Cellular Contractile Function In Vitro
}

\author{
Alec S.T. Smith ${ }^{1}$, Christopher J. Long ${ }^{1}$, Christopher McAleer ${ }^{1}$, Nathaniel Bobbitt ${ }^{1}$, Balaji Srinivasan ${ }^{1}$, James J. Hickman ${ }^{1}$ \\ ${ }^{1}$ NanoScience Technology Center, University of Central Florida
}

Correspondence to: James J. Hickman at jhickman@mail.ucf.edu

URL: http://www.jove.com/video/51866

DOI: doi: $10.3791 / 51866$

Keywords: Bioengineering, Issue 92, cantilever, in vitro, contraction, skeletal muscle, NMJ, cardiomyocytes, functional

Date Published: 10/3/2014

Citation: Smith, A.S., Long, C.J., McAleer, C., Bobbitt, N., Srinivasan, B., Hickman, J.J. Utilization of Microscale Silicon Cantilevers to Assess Cellular Contractile Function In Vitro. J. Vis. Exp. (92), e51866, doi:10.3791/51866 (2014).

\section{Abstract}

The development of more predictive and biologically relevant in vitro assays is predicated on the advancement of versatile cell culture systems which facilitate the functional assessment of the seeded cells. To that end, microscale cantilever technology offers a platform with which to measure the contractile functionality of a range of cell types, including skeletal, cardiac, and smooth muscle cells, through assessment of contraction induced substrate bending. Application of multiplexed cantilever arrays provides the means to develop moderate to high-throughput protocols for assessing drug efficacy and toxicity, disease phenotype and progression, as well as neuromuscular and other cell-cell interactions. This manuscript provides the details for fabricating reliable cantilever arrays for this purpose, and the methods required to successfully culture cells on these surfaces. Further description is provided on the steps necessary to perform functional analysis of contractile cell types maintained on such arrays using a novel laser and photo-detector system. The representative data provided highlights the precision and reproducible nature of the analysis of contractile function possible using this system, as well as the wide range of studies to which such technology can be applied. Successful widespread adoption of this system could provide investigators with the means to perform rapid, low cost functional studies in vitro, leading to more accurate predictions of tissue performance, disease development and response to novel therapeutic treatment.

\section{Video Link}

The video component of this article can be found at http://www.jove.com/video/51866/

\section{Introduction}

The in vitro culture of muscle cells from both human and rodent sources has been possible for decades ${ }^{1,2}$. However, while standard coverslip preparations are useful for biochemical assessment, they do not facilitate analysis of the cell's primary functional output (contractility), and therefore are of somewhat limited value as a means to assess cellular maturation and performance. In order to maximize the amount of data obtainable from such in vitro cultures, it is necessary to advance the development of systems capable of housing such cells in configurations that permit the real-time assessment of their functional performance. The establishment of a multitude of three dimensional muscle models has made some progress toward fulfilling this need, and such systems have been used in a number of publications as a means to assess the contractile capacity of cultured muscle cells in vitro ${ }^{3-5}$. While such systems are invaluable for tissue modeling and reconstruction studies, they are limited in their applicability for studies of single cell responses. In such cases where single fiber studies are necessary, complex and labor intensive ex vivo methodologies remain the only option ${ }^{6-10}$. Furthermore, current movement toward the development of complex, multi-organ platforms for drug development and screening protocols requires the establishment of systems which are non-invasive, easily scalable and which integrate readily with supporting cells and tissue models ${ }^{11}$

Microscale cantilevers offer a simple method for assessing the functional contractile capacity of single cells/small populations of cells ${ }^{12,13}$. The technique is based on modified Atomic Force Microscopy (AFM) technology ${ }^{14}$, and uses a laser and photo-detector system to measure microscale cantilever deflection in response to cultured myotube contractile activity. Modified Stoney's equations are then used to calculate stress in the myotube, and the force exerted by the myotube in order to generate the observed substrate deflection ${ }^{15}$. A scanning program has been written which enables simultaneous assessment of multiplexed cantilever arrays, offering potential moderate to high through-put applications for drug toxicity/efficacy studies ${ }^{15,16}$. Such technology may prove invaluable in the development of functional, pre-clinical assays for predicting drug efficacy in vivo. Furthermore, fabrication of cantilever chips in silicon does not impede post analysis processing of cells for standard biomolecular assays such as immunostaining, western blotting and PCR.

This manuscript provides detailed instructions on the fabrication and preparation of microscale silicon cantilevers, the hardware and software set-up, and the operating guidelines for assessing the functionality of contractile cells cultured on these chips. Standard cell culture techniques can be implemented for plating and maintenance of cells on these surfaces, hence any contractile cell type for which reliable culture parameters exist should be able to integrate with this device with ease. The relatively simple 2D culture parameters utilized in this system makes integration of other cell models or addition of cell types that can interact with muscle (such as innervating neurons) straight-forward, greatly increasing the applicability of this model in the development of more complex functional in vitro assays and multi-organ models of mammalian systems. 


\section{Cantilever Chip Fabrication}

Illustrated details of the described fabrication steps are provided in Figure 1.

1. Place Silicon-On-Insulator (SOI) wafers in an oven and bake at $125^{\circ} \mathrm{C}$ for 20 min to dehydrate them.

2. Deposit a $1.5 \mu \mathrm{m}$ thick layer of silicon oxide onto the handle layer of the dehydrated SOI wafer using a Plasma Enhanced Chemical Vapor Deposition (PECVD) tool.

3. Place the wafer on the spin coater chuck with the device layer facing up. Ensure wafer is centered, dispense $2 \mathrm{ml}$ of P20 primer on the center of the wafer, and spin at $3,000 \mathrm{rpm}$ for $60 \mathrm{sec}$ at an acceleration of $1,000 \mathrm{rpm} / \mathrm{sec}$. P20 primer promotes the adhesion of photoresist to the device layer.

4. While the wafer is still on the spin coater chuck, dispense $2 \mathrm{ml}$ of $\mathrm{S} 1818$ photoresist on the center of the wafer, and spin at 3,000 rpm for 60 $\mathrm{sec}$ at an acceleration of $1,000 \mathrm{rpm} / \mathrm{sec}$ to obtain a $1.5 \mu \mathrm{m}$ thick layer of photoresist.

5. Place the wafer on a hot plate and perform a soft bake at $115^{\circ} \mathrm{C}$ for $1 \mathrm{~min}$.

6. Perform a hard contact UV exposure using a photolithography contact mask aligner in order to transfer the pattern from the cantilever mask to the photoresist on the device layer. Calculate the exposure time based on exposure energy value of $125 \mathrm{~mJ} / \mathrm{cm}^{2}$ for $\mathrm{S} 1818 \mathrm{photoresist}$.

7. Develop the photoresist by immersing the wafer in a 726 MIF photoresist developer for 1 min while gently shaking the wafer back and forth.

8. Rinse the wafer with de-ionized (DI) water and dry it, preferably using a spin rinse dryer (SRD) tool.

9. Remove the photoresist from the edge of the wafer (up to $5 \mathrm{~mm}$ from the edge) using a cotton swab dipped in acetone.

10. Load the wafer in a Deep Reactive Ion Etch (DRIE) tool, with the photoresist side facing up, and run a recipe to etch the patterned silicon layer through the device layer. The buried oxide layer functions as an etch stop, so perform an etch with $50 \%$ to $100 \%$ more cycles than is expected to be necessary to ensure a complete etch.

11. Place the wafer in a hot photoresist bath solution for $20 \mathrm{~min}$ to strip the photoresist. Transfer the wafer to a quick-dump-rinser (QDR) to rinse the wafer with DI water. Following the photoresist strip, load the wafer in a SRD tool to rinse and dry the wafer. Inspect the wafer under a microscope to ensure the cantilever pattern appears as expected.

12. Deposit a $1 \mu \mathrm{m}$ thick layer of un-doped silicon oxide on the device layer of the wafer using a PECVD tool. This oxide layer functions as a protective layer for the cantilevers during further processing steps.

13. Place the wafer on the spin coater chuck with the handle layer facing up to begin processing of the back side of the wafer. Ensure wafer is centered, dispense $2 \mathrm{ml}$ of P20 primer on the center of the wafer, and spin at 3,000 rpm for $60 \mathrm{sec}$ at an acceleration of 1,000 rpm/sec.

14. While the wafer is still on the spin coater chuck, dispense $2 \mathrm{ml}$ of SPR220-4.5 photoresist onto the center of the wafer, and spin at $2,000 \mathrm{rpm}$ for $45 \mathrm{sec}$ at an acceleration of $1,000 \mathrm{rpm} / \mathrm{sec}$ to obtain a $6.5 \mu \mathrm{m}$ thick layer of photoresist.

15. Place the wafer on a proximity hot plate to perform a soft bake at $115^{\circ} \mathrm{C}$ for $2 \mathrm{~min}$.

16. Using a photolithography contact aligner capable of front/back alignment, align the backside window mask to the front side cantilever pattern and perform a hard contact UV exposure in order to transfer the pattern from the backside window mask to the photoresist on the handle layer. Use an exposure time calculated based on exposure energy value of $480 \mathrm{~mJ} / \mathrm{cm}^{2}$ for SPR220-4.5 photoresist.

17. After UV exposure, let the wafers remain in a dark area for $30 \mathrm{~min}$ before the next processing step.

18. Develop the photoresist by immersing the wafer in a 726 MIF photoresist developer for 2 min while gently shaking the wafer back and forth.

19. Rinse the wafer with de-ionized (DI) water and dry it, preferably using a spin rinse dryer (SRD) tool.

20. Place the wafers in an oven at $90{ }^{\circ} \mathrm{C}$ for $12 \mathrm{hr}$.

21. Etch the silicon oxide layer on the backside of the wafer using an RIE system with fluorinated gases and a recipe for etching oxide. The patterned oxide on the backside of the wafer acts as an etch mask for further processing. Inspect the wafers under a microscope to ensure that the silicon oxide in the exposed window is completely etched.

22. Remove the photoresist on the edge of the wafer (up to $5 \mathrm{~mm}$ from the edge) using a clean room swab dipped in acetone.

23. Load the wafer in a DRIE tool with and run a recipe to etch the patterned handle layer to a depth of $500 \mu \mathrm{m}$ with the buried oxide layer functioning as an etch stop. Split this etch into multiple runs to prevent excessive heating of the wafer, which causes inconsistent etching of the silicon. This etch step completely removes the silicon underneath the cantilevers.

24. Perform a wet etching step using $25 \%$ dilute HF etchant, to strip the buried oxide layer below the cantilevers and the protective oxide layer on top of the cantilevers.

NOTE: This step releases the bottom surface of the silicon cantilevers and also opens a window underneath to provide an access to probe the cantilever with the laser.

25. Rinse the wafer using a series of DI water baths and carefully dry with nitrogen. Since the cantilevers are supported only at their bases, do not use forceful sprays of DI water or inert gas directly on the cantilevers.

26. Cleave the individual chips from the wafer along the cleave lines produced during the handle layer etch step.

\section{Cell Culture}

1. Prepare $13 \mathrm{~F}$ coverslips according to previously published methods ${ }^{17}$.

NOTE: If $13 \mathrm{~F}$ coverslips are not available, any hydrophobic surface with a contact angle above $95^{\circ}$ can be used.

2. Sterilize cantilever chips and $13 \mathrm{~F}$ coverslips in a $70 \%$ ethanol solution and allow to air dry in a flow hood.

3. Place individual cantilever chips on top of $13 \mathrm{~F}$ coverslips inside a standard 12 well plate.

4. Coat the cantilevers with the biopolymer or surface modification optimized for the cell type being used according to the standard cell culture protocols.

5. Re-suspend the cells in their specific growth medium to the desired concentration.

NOTE: This protocol will result in a substantial number of cells falling through the cantilever window and not adhering to the desired cantilever surface. Cell preparations should therefore be made $3-4 x$ more concentrated than for standard coverslip preparations. For example, seeding of rat skeletal muscle satellite cells is typically carried out using a seeding density of 500 to 700 cells/square mm ${ }^{15,16}$, and are used with 
cantilever substrates at 2,000 cells/square $\mathrm{mm}$. Optimization experiments should be carried out with new cell sources to ascertain an appropriate seeding density.

6. Pipette $200 \mu \mathrm{l}$ of the cell suspension onto the cantilever chip surface, ensuring the bubble of medium covers the cantilever windows entirely. If the medium wicks through the window and doesn't form a static bubble on top of the cantilevers replace the 13F coverslip and reattempt the plating.

7. Transfer the plate containing the chips to an incubator and allow the cells to adhere for at least $1 \mathrm{hr}$ (preferably 2-3 hr).

8. After this plating period, use sterile forceps to transfer the chip to a clean well, without a 13F coverslip, and top up the culture with $1 \mathrm{ml}$ of growth medium.

9. Return the plate to the incubator.

10. Maintain cells according to their standard protocol for in vitro maintenance on coverslips. For skeletal muscle cells, a switch of medium composition to induce myotube formation once cells become confluent will be necessary.

\section{Setup of Hardware and Software for Cantilever Deflection Analysis}

1. Place a heated culture dish into the stage of an upright electrophysiology microscope.

2. Add $3 \mathrm{ml}$ of the feeding medium the cells are currently suspended in (+ $10 \mathrm{mM} \mathrm{HEPES})$ to the heated microscope stage.

3. Mount stainless steel electrodes on the inside of the heated culture dish at a separation distance of $15 \mathrm{~mm}$ and connect them to a pulse generator, capable of producing field stimulation pulses of varying intensity, frequency, and waveform, to allow the system to produce field stimulation of cells when appropriate.

4. Bolt a Helium-Neon laser, mounted on XY translational stages, to the underside of the microscope table and adjust it so that the laser beam is directed through the base of the heated culture dish at a $30^{\circ}$ angle relative to the plane of the cantilever.

5. Bolt a quadrant photo-detector module, mounted on $X Y$ translational stages, to the underside of the microscope stage and adjust the position so that the reflected laser beam lands in the center of the 4 quadrants. Figure 2 provides an overview of the hardware set up necessary for implementing the described protocol.

6. Write a software program to control the linear actuators that scan across the cantilevers. Write the software program with reference to the flow chart provided in Figure 3. The graphical interface programmed for use with this system is provided in Figure 4.

\section{Recording Cantilever Deflection Data}

1. Turn on the cantilever analysis hardware and associated software.

2. Insert the heated stage thermistor into the medium and wait for it to read $37^{\circ} \mathrm{C}$.

3. Insert the cantilever chip into the stage with the cantilevers oriented toward the right hand side of the stage.

4. Turn on the microscope light source.

5. Focus the microscope to bring the edges of the cantilevers into view and use the laser/photo-detector control software to position the laser beam on the tip of cantilever 1. NOTE: Assuming the cantilevers are oriented to the right of the stage, cantilever 1 is the one positioned in the top left of the array and numbers run down to 16 in the bottom left. Cantilever 17 is in the top right position and runs to 32 in the bottom right (Figure 5A).

6. Press "play" on the recording software.

7. Position the photo-detector so that the signal reads " 0 " in both $\mathrm{x}$ and $\mathrm{y}$ frames by adjusting the stepper motors controlling the photo-detector.

8. Set cantilever 1 position in the laser/photo-detector control software (Figure 4).

9. Move the laser to the tip of cantilever 16, repeat step 4.7., and set cantilever 16 position in the laser/photo-detector control software.

10. Move the laser to the tip of cantilever 32, repeat step 4.7., and set cantilever 32 position in the laser/photo-detector control software.

11. Move the laser to the tip of cantilever 17, repeat step 4.7., and set cantilever 17 position in the laser/photo-detector control software.

12. Turn off the microscope light source and the overhead light in the laboratory.

13. Press "record" on the recording software.

14. Set the pulse generator hardware to $40 \mathrm{msec}, 5 \mathrm{~V}$ pulses at a frequency of $1 \mathrm{~Hz}$, and turn the machine on. NOTE: Optionally, employ optimized stimulation protocols for specific cell sources at this point, the stated settings are guidelines based on collected data using human and rodent cell sources ${ }^{12,13,15,16}$.

15. Using the laser/photo-detector control software, set the hardware to scan across the 32 cantilever array, stopping for 5 sec at each one.

16. When the scan of the 32 cantilevers is complete, turn off the stimulator, then stop the recording software and bring up the data file.

17. Examine the recorded trace from each cantilever for evidence of contractile activity. Make a note of each cantilever with positive responses. A contraction is defined as a peak if the deflection is at least $0.1 \mathrm{~V}$ above the baseline.

18. Remove any non-responsive cantilevers from the scan protocol on the laser/photo-detector control software.

19. The active cantilevers can then be rescanned without stimulation in order to get a reading of the cell's spontaneous contractile activity.

20. Run scans with or without broad field electrical stimulation, following addition of a therapeutic compound to the medium in order to observe its effect on the functional output of the cultured cells.

21. Carry out fatigue assessments by electrically stimulating the cells for extended periods and scanning levels of contractility to measure how long it takes for peak force to drop below a specific threshold.

22. In experiments where motor neurons are maintained in co-culture with muscle, measure neuromuscular junction formation through treatment of motoneuron-myotube cantilever co-cultures with a neuronal stimulant (such as glutamate) or synaptic inhibitor (e.g., D-tubocurarine) and scanning for increases and decreases in spontaneous activity respectively ${ }^{16}$

23. Perform specific scans as dictated by the needs of the planned experiments.

\section{Analysis of Cantilever Deflection Data}

1. Use modified Stoney's equations ${ }^{15}$ (detailed below) to convert raw cantilever deflection data (in Volts) into a readout of stress in the cell layer or myotube (in Pascals), and a direct measurement of cellular contractile force (in nano-Newtons) : 


$$
\begin{aligned}
\delta & =\frac{2 L}{3} \tan \left[\frac{\theta}{2}-\frac{1}{2} \arctan \left(\tan \theta-\frac{\text { Voltage }}{C_{\text {detector } \times P \times \cos \theta}}\right)\right] \text { Equation } 1 \\
\sigma_{c} & =\frac{E_{S i} t_{S i}^{3}}{6 t_{f}\left(1-v_{S i}\right)\left(t_{f}+t_{S i}\right)} \frac{3 \delta}{2 L^{2}} \times \frac{1}{1+\frac{t_{f}}{t_{S i}}} \text { Equation 2 }
\end{aligned}
$$

2. Where $\delta=$ cantilever tip deflection and stress produced by the myotube, $\sigma_{c}=$ stress produced by the myotube, assuming a uniform thick film the full width of the cantilever, $C_{\text {detector }}=$ the system-specific coefficient relating voltage to laser position on the photo-detector, $\theta=$ the angle of the laser and detector relative to the plane of the cantilever, $E_{S i}=$ the elastic modulus of silicon, $t_{S i}=$ the thicknesses of the cantilever, $t_{f}=$ myotube thickness, $v_{S i}=$ poison's ratio of silicon, $L=$ cantilever length, $P=$ path length of laser from cantilever tip to detector, and $w_{S i}=$ the width of the cantilever. A schematic explaining the terms used in these equations is provided in Figure 6.

3. Assuming the myotube is a uniform film, the force in the myotube is equal to the force in the film, leading to Equation 3 , by equating the calculation of force from stress and the assumed cell cross sectional area that was used for the application of Stoney's equation. $F_{\text {myotube }}=\sigma_{c} \times t_{f} \times w_{S i}$ Equation 3

4. Following functional data collection, fix cantilever chips for immunocytochemical analysis or utilize cells for protein or DNA analysis using standard techniques.

5. Optionally, return the cells to the incubator instead of preparing for molecular analysis in order to reassess functional performance at a later time-point.

\section{Representative Results}

Successful culture of contractile cells on cantilevers is a relatively straightforward procedure, utilizing standard cell culture techniques (Figure 5). The percentage of cantilevers supporting contracting cells will vary depending on cell type being examined and specific culture technique employed. Using primary embryonic cells derived from rat hind limbs, contractile activity was detected on $12 \%$ of cantilevers examined $(n=4)$. Analysis of contractile function using the laser and photo-detector system described provides accurate real-time data pertaining to the functional maturity of the seeded cells. Use of standard electrophysiological software can then be used to analyze the raw data, facilitating calculation of relevant functional properties, such as peak force, time to peak force, and time to half relaxation, as illustrated in Figure 7 . Subsequent data collection from cultures treated with therapeutic compounds allows for comparison of functional properties with and without drug addition, thereby enabling assessment of compound activity and subsequent prediction of in vivo responses. Furthermore, extended stimulation protocols provide the means to assess rates of fatigue in cultured cells, thus broadening the level of physiological data obtainable from this system (Figure 8).

The cantilever culture can be modified to include motor neurons in the culture system with skeletal muscle myotubes ${ }^{16}$, so as to allow assessment of neuromuscular synapse formation in vitro (Figure 9). In such cultures, rates of spontaneous contractile activity are compared to rates of contraction in response to treatment with a neuron-specific stimulant, such as glutamate. Any observed glutamate induced increases in contraction rates suggest the activation of cultured neurons, leading to acetylcholine release and subsequent myotube activation. Treatment with synaptic inhibitors, such as the acetylcholine receptor blocker D-tubocurarine, leading to cessation of glutamate induced activity provide further evidence for the presence of functional neuromuscular synapses in these cultures ${ }^{16}$. 


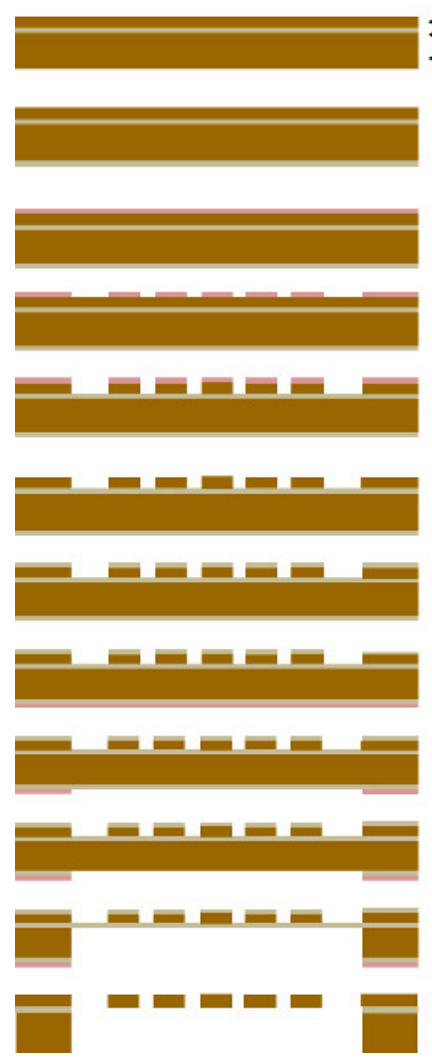

$\longleftarrow$ Device Layer ( $4 \mu \mathrm{m})$ ( $1 \mu \mathrm{m}$ )

$\leftarrow$ Handle Layer $(500 \mu \mathrm{m})$

2. $\mathrm{SIO}_{2}$ deposition on handle layer

3. Spin coat photoresist

6. Develop photoresist

7. DRIE Si etch

10. Strip photoresist

11. Deposit protective layer of oxide

12. Spin coat photoresist

15. Develop photoresist

17. Etch oxide

19. DRIE si etch

20. Strip oxide layers to release cantilevers
Silicon Dioxide

Silicon

Photoresist

Figure 1. Schematic detailing cantilever fabrication process flow. The numbers listed in the flow diagram correlate to protocol steps in the methods section titled "Cantilever chip fabrication." Please click here to view a larger version of this figure. 



Figure 2. Hardware details of the laser/photo-detector system used to assess cellular contraction on cantilevers. (A) Photograph of the modified electrophysiological microscope used for cantilever assessment. The laser (i) and photo-detector (ii) are mounted beneath the stage on $\mathrm{XY}$ translational stages. A transparent culture dish is mounted on the stage (iii), which allows laser passage to the cantilever array through the underside of the stage. (B) Schematic "top-down" perspective of the microscope stage. XY translational stages allow movement of the laser and photo-detector in both $X$ and $Y$ planes (red arrows), facilitating movement of the laser to interrogate each cantilever in an array. Cantilever chips (iii) should be placed into the stage with the cantilevers facing toward the right of the stage in order for the system to operate correctly. (C) Close up photograph of the laser (i) and photo-detector (ii) mounted beneath the microscope stage. The laser is positioned at a $30^{\circ}$ angle relative to the plane of the cantilever chip $(\theta)$. (D) Close up photograph of the culture dish. Broad-field electrical stimulation is applied by means of a pair of silver electrodes positioned $15 \mathrm{~mm}$ apart (iv). A heating element $(\mathrm{v})$ attached to the underside of the dish is used to maintain the culture at $37^{\circ} \mathrm{C}$ during analysis. Temperature control is dynamic, and regulated by means of a thermistor placed in the medium (not shown). Please click here to view a larger version of this figure.



Figure 3. Flow chart of logic underlying software to control scanning of the laser and photo-detector. Two software loops control motion: a main loop for user input and a loop that controls the scanning motion. Setup is performed while in the main loop, followed by activation of the second loop for medium- to high-throughput data collection. Please click here to view a larger version of this figure. 


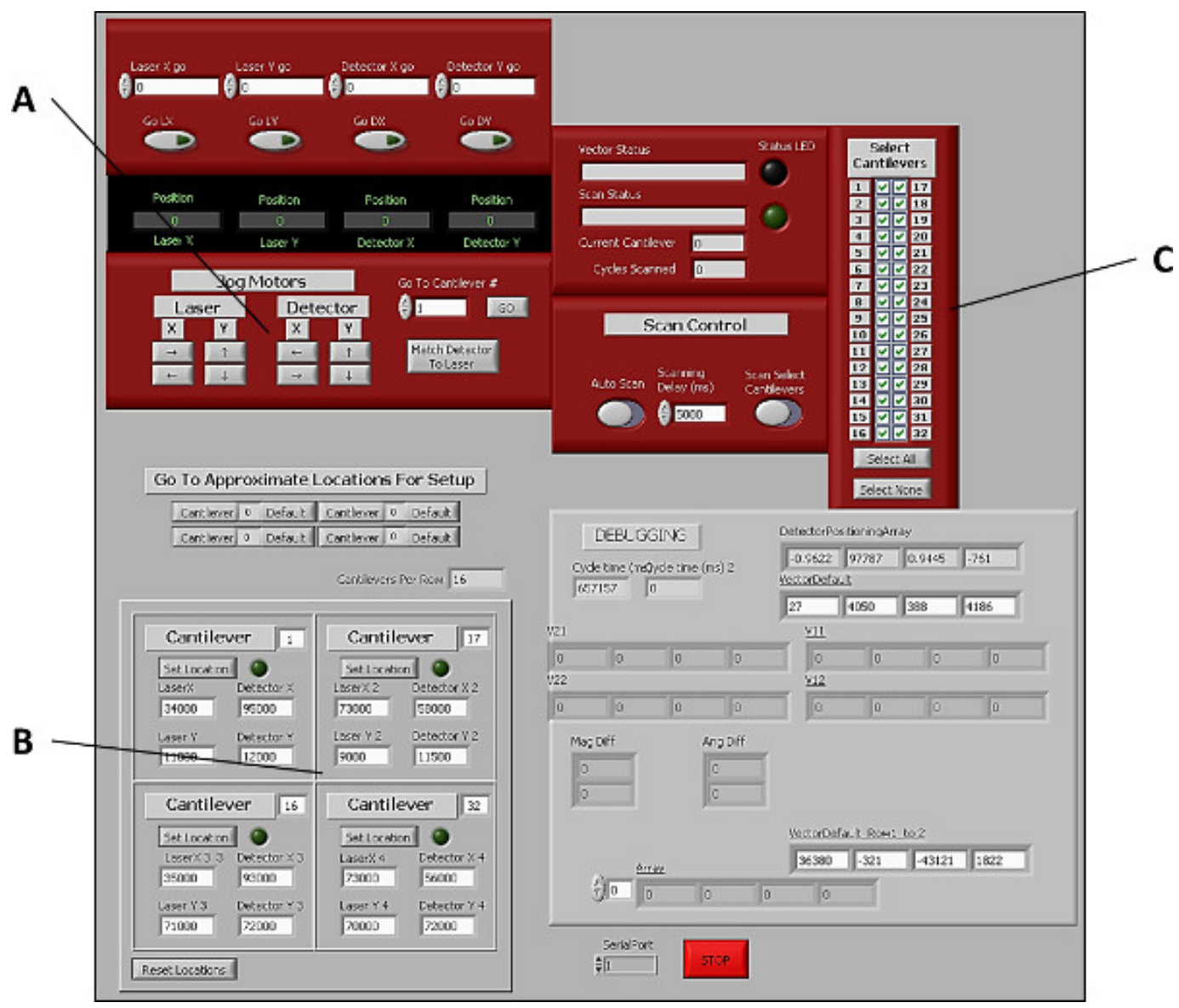

Figure 4. Graphical user interface of software used to control laser and photo-detector positions during cantilever assessment. (A) Buttons to manually control laser and photo-detector positions in both $X$ and $Y$ planes. (B) Controls for manually setting the positions of the 4 corner cantilevers $(1,16,17$, and 32$)$, thereby allowing the software to extrapolate the positions of the remaining cantilevers in the array. (C) Controls allowing users to select which cantilevers to include in each scan. Please click here to view a larger version of this figure. 



Figure 5. Images of a custom built cantilever chip for use in this system, and representative images of cells maintained on similar surfaces. (A) Photograph of a single, custom built cantilever chip. Each chip contains 32 cantilevers arranged in 2 rows of 16 . Cantilever 1 is positioned in the bottom left of the image and cantilever 32 in the top right. Cantilever chips are $15 \times 15 \mathrm{~mm}^{2}$. (B) Phase-contrast image of primary rat skeletal muscle cells grown on cantilevers. Each cantilever is $750 \mu \mathrm{m}$ long, $100 \mu \mathrm{m}$ wide and $4 \mu \mathrm{m}$ thick. (C) Immunocytochemical stain of a primary rat myotube maintained on a cantilever. Cells were stained using an antibody probe for Myosin Heavy Chain. Note the striated appearance of the cultured fiber, indicating maturation of the contractile machinery. Cantilever edges have been artificially highlighted in this image to provide an indication of scale. Please click here to view a larger version of this figure.



Figure 6. Schematic illustrating the terms used in Stoney's equations for deriving force produced by myotubes on cantilevers. $\delta$ $=$ cantilever tip deflection and stress produced by the myotube, $C_{\text {detector }}=$ the system-specific coefficient relating voltage to laser position on the photo-detector, $\theta=$ the angle of the laser and detector relative to the plane of the cantilever, $E_{S i}=$ the elastic modulus of silicon, $t_{S i}=$ the thicknesses of the cantilever, $t_{f}=$ myotube thickness, $v_{S i}=$ poison's ratio of silicon, $L=$ cantilever length, and $P=$ path length of laser from cantilever tip to detector. Please click here to view a larger version of this figure. 

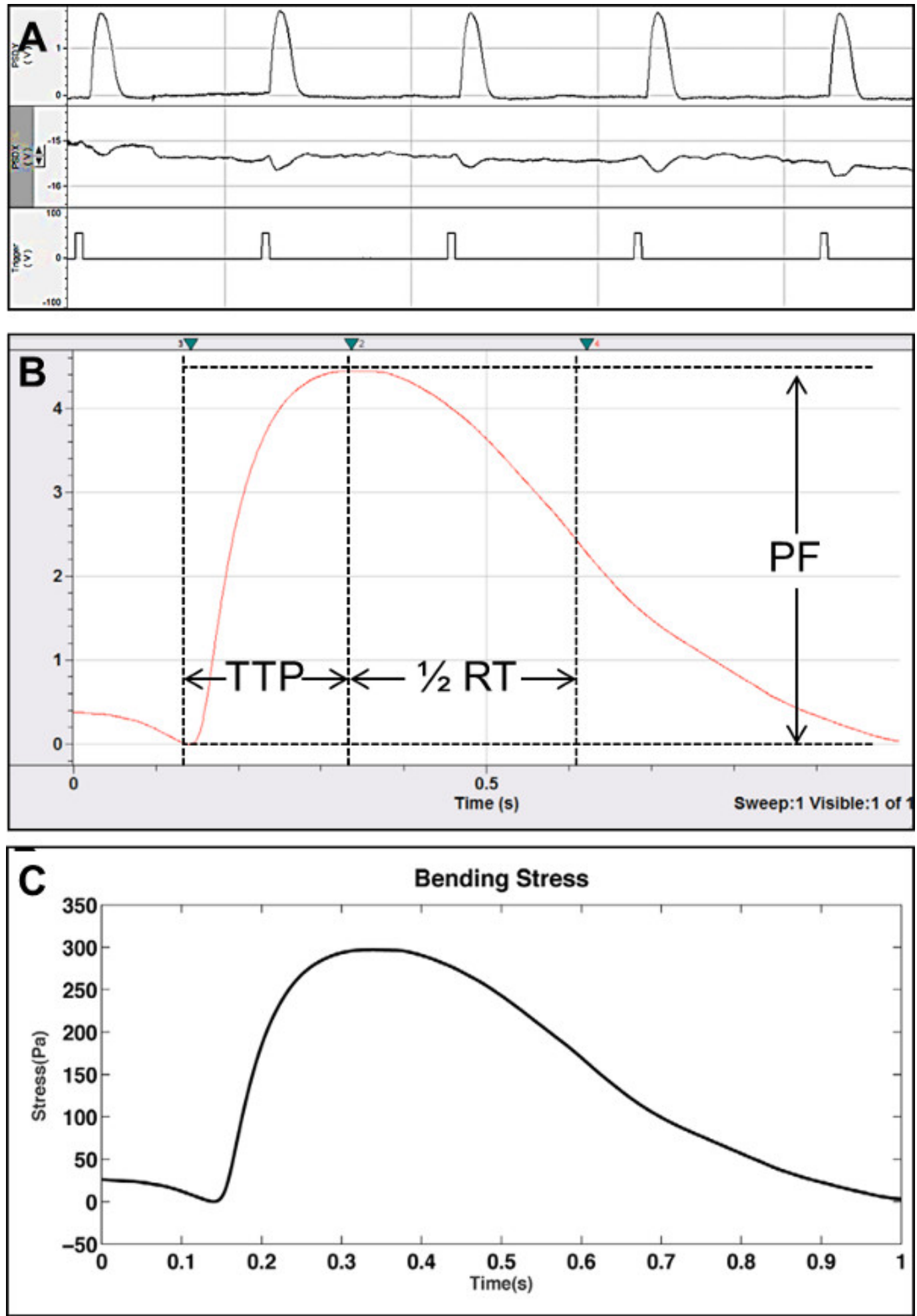

Figure 7. Representative raw data and analysis of contractile form using the cantilever system described. (A) Example of a raw data trace from the broad-field electrical stimulation of primary rat myotubes on cantilevers. Top trace $=$ laser deflection (in Volts) in the $x$ axis, indicating lengthwise strain on the cantilever. Middle trace = laser deflection (in Volts) in the $y$-axis, indicating torsional strain across the cantilever. Bottom trace = Indication of the temporal position of electrical pulses used to elicit myotube contraction in this system. (B) Using standard electrophysiology software, it is possible to measure peak force (PF), time to peak force (TTP) and time to half relaxation (1/2 RT) from the collected raw data. (C) Collated contraction data $(n=11)$ from primary rat skeletal muscle myotubes. Application of a modified Stoney's equation allows calculation of cantilever stress from raw data readings in Volts. From this data, direct calculation of force (in Newtons) can also be generated. Published data reprinted with permission ${ }^{13}$. Please click here to view a larger version of this figure. 




Figure 8. Representative data demonstrating analysis of skeletal muscle fatigue in cantilever cultures. Raw data (in Volts) was converted to a measurement of myotube force (in nano-Newtons) and re-plotted. Data illustrates magnitude of myotube contractions on cantilevers in response to $1 \mathrm{~Hz}$ broad-field electrical pulses after $0 \mathrm{~min}$ (black trace) and $120 \mathrm{~min}$ (grey trace) of continuous stimulation. Please click here to view a larger version of this figure.

A



B



C



Figure 9. Representative traces from the analysis of a skeletal muscle-motoneuron co-culture maintained on cantilevers, demonstrating the functional effects of motoneuron stimulation with and without addition of a neuromuscular blocker. Raw data (in Volts) was converted to a measurement of myotube force (in nano-Newtons) and re-plotted. (A) Measurement of spontaneous contractions by the cultured myotubes without neuronal stimulation. (B) Measurement of myotube contraction following neuronal stimulation via the addition of $200 \mu \mathrm{M}$ glutamate. (C) Measurement of myotube contraction following glutamate and $12.5 \mu \mathrm{M}$ D-tubocurarine treatment. Published data reprinted with permission ${ }^{16}$. Please click here to view a larger version of this figure.

The following tables list the specific reagents used to culture cells in the validation experiments described, as well as the equipment necessary to perform the cantilever assessment. Please note that culture reagents used are not necessary and this system should be easily integrated 
with the culture protocols of any lab maintaining contractile cells in vitro. The listed reagents are provided should investigators wish to repeat the specific experimental results described.

Discussion

The critical steps in analyzing microscale cantilevers for evidence of cellular contraction are the placement of the cantilever chip within the microscope stage, and the subsequent alignment of the laser and photo-detector with the tip of the corner cantilevers in the array. If this is not done accurately, then the software will be unable to extrapolate the positions of the remaining cantilevers in the array, potentially leading to accumulation of false negatives during data collection. Operators should take care to ensure that the cantilever chip is lying flush with the bottom of the culture dish before calibrating the laser positions. If the chip sits at an angle in the dish, it will alter the path of the deflected laser beam and confound data collection.

A functional air-table must be employed to cancel out background vibrations during data collection. The small thickness (roughly $4 \mu \mathrm{m}$ ) of the cantilevers used in this study provides high sensitivity to contractile activity, but has the side effect of increased sensitivity to vibrations. Operators should take care to move around the hardware as little as possible during data recording, to reduce background readings. Finally, when placing the cantilever chip in the stage, take care to ensure the chip does not come into contact with the stimulating silver electrodes in the culture well. Application of broad-field stimulation with the electrode touching the chip will alter the current path and negatively impact on the system's ability to effectively stimulate the cultured cells.

In order to obtain more accurate readings of force output in skeletal muscle myotubes, it is highly recommended to perform immunostaining of the cultured cells once functional analysis is complete. The equations listed require input of a myotube's cross-sectional area (CSA) in order to calculate force. While an assumed value can be used based on previous data, measurement of a myotube's exact CSA using confocal imaging techniques will provide a more accurate estimation of the force exerted by the contracting myotube. Relating raw contraction data to the physical characteristics of the cell in question is valuable in normalizing results across multiple cantilevers and between experimental conditions ${ }^{15}$, and therefore critical for generating accurate predictions of whole tissue responses to drug treatment or disease. Stoney's equations assume the myotubes examined span the entire length of the cantilever so effort should be made to only include data obtained from cells which comply with this assumption. If this is not possible, finite element analysis can be used to provide an accurate measurement of force from smaller myotubes as published previously ${ }^{15}$. This analytical method is far more labor intensive, and therefore ill-suited to higher throughput applications, but may be necessary if optimized cell cultures conforming to Stoney's assumptions cannot be obtained.

As already stated, culture parameters can be transferred from standard coverslip preparations. However, it is advised to consider utilizing serumfree media compositions and non-biological substrates for use of this system in drug screening applications. Due to the undefined nature of animal sera, the effect of novel therapeutics may be confounded by the inclusion of such additives in the culture medium. Serum-free media formulations are available for both rodent and human skeletal muscle cultures ${ }^{18-21}$ and provide a more controlled, well defined environment for performing compound assessment. Similarly, the use of biological coatings (collagen, laminin, etc.) on cantilevers introduces variability to the cell preparations which are avoided by application of non-biological substrates. The deposition of silane self-assembled monolayers onto culture surfaces have been shown extensively to support the adherence and development of multiple cell types ${ }^{16,18,20,22-31}$, and represent the means to generate fully-defined surfaces in a reliable and repeatable manner.

Due to the range of contractile cells present in mammalian systems, the application of this model for in vitro assays is relatively broad. Although optimized using skeletal muscle cells, the system is potentially applicable to smooth muscle cells and cardiomyocytes as well, providing the means to perform the rapid assessment of dose responses to novel therapeutics in these cells in real-time. Current cantilever chips consist of an array of 32 cantilevers (Figure 5A), but could be easily altered to include many more. Analysis of such arrays produces a substantial number of data points from a single culture, thereby greatly increasing the statistical power of any observed differences. As evidenced in Figure 8, cells cultured in this system undergo fatigue over time, in response to continuous broad-field stimulation, allowing assessment of drug effects on the endurance levels of cells in vitro. Addition of innervating motor neurons to this culture model also allows assessment of synapse formation through measurement of muscle contraction in response to neuronal stimulants (Figure 9). While the measurement of baseline functional parameters in a multiplexed assay (Figure 7) has obvious advantages over standard biomolecular assessment of in vitro cultures, the ability to assess neuromuscular functionality and the endurance capacity of seeded cells greatly increases the applicability of this model for drug screening and disease modeling applications. The described technique offers investigators the means to perform rapid, low cost functional assessment of both healthy and diseased muscle cells in vitro. The versatility of the culture system, and the high level of functional detail obtainable from analysis of the raw data, should produce more accurate predictions of in vivo tissue responses to novel pathological and chemical challenges.

\section{Disclosures}

The authors have nothing to disclose.

\section{Acknowledgements}

This research was funded by National Institute of Health grant numbers R01NS050452 and R01EB009429. Fabrication of the cantilever chips was performed externally by collaborators at the NanoFabrication Facility located at Cornell University. All equipment used in the cantilever fabrication process was located in this facility. Special thanks to Mandy Esch and Jean-Matthieu Prot for their assistance with cantilever microfabrication. Video animation of cantilever functionality was generated by Charles Hughes, Alex Zelenin and Eric Imperiale from the Synthetic Reality Lab at UCF. 


\section{References}

1. Bischoff, R. Enzymatic liberation of myogenic cells from adult rat muscle. Anat. Rec. 180, 645-661, doi:10.1002/ar.1091800410 (1974)

2. Yasin, R. et al. A quantitative technique for growing human adult skeletal muscle in culture starting from mononucleated cells. J. Neurol. Sci. 32, 347-360 (1977).

3. Dennis, R. G., \& Kosnik, P. E., 2nd. Excitability and isometric contractile properties of mammalian skeletal muscle constructs engineered in vitro. In Vitro Cell Dev. Biol. Anim. 36, 327-335, doi:10.1290/1071-2690(2000)036<0327:eaicpo>2.0.co;2 (2000).

4. Khodabukus, A., \& Baar, K. Defined electrical stimulation emphasizing excitability for the development and testing of engineered skeletal muscle. Tissue Eng Part C Methods. 18, 349-357, doi:10.1089/ten.TEC.2011.0364 (2012).

5. Langelaan, M. L. P. et al. Advanced maturation by electrical stimulation: Differences in response between C2C12 and primary muscle progenitor cells. Journal of tissue engineering and regenerative medicine. 5, 529-539, doi:10.1002/term.345 (2011).

6. Stephenson, G. M., O'Callaghan, A., \& Stephenson, D. G. Single-fiber study of contractile and biochemical properties of skeletal muscles in streptozotocin-induced diabetic rats. Diabetes. 43, 622-628 (1994).

7. Harber, M., \& Trappe, S. Single muscle fiber contractile properties of young competitive distance runners. Journal of applied physiology (Bethesda, Md. : 1985). 105, 629-636, doi:10.1152/japplphysiol.00995.2007 (2008).

8. Hvid, L. G. et al. Four days of muscle disuse impairs single fiber contractile function in young and old healthy men. Experimental gerontology. 48, 154-161, doi:10.1016/j.exger.2012.11.005 (2013).

9. Edman, K. A. Contractile performance of striated muscle. Advances in experimental medicine and biology. 682, 7-40, doi:10.1007/978-1-4419-6366-6_2 (2010).

10. Krivickas, L. S., Walsh, R., \& Amato, A. A. Single muscle fiber contractile properties in adults with muscular dystrophy treated with MYO-029. Muscle Nerve. 39, 3-9, doi:10.1002/mus.21200 (2009).

11. Sung, J. H. et al. Microfabricated mammalian organ systems and their integration into models of whole animals and humans. Lab on a chip. 13, 1201-1212, doi:10.1039/c3lc41017j (2013).

12. Wilson, K., Molnar, P., \& Hickman, J. J. Integration of functional myotubes with a Bio-MEMS device for non-invasive interrogation. Lab on a chip. 7, 920-922 (2007).

13. Wilson, K., Das, M., Wahl, K. J., Colton, R. J., \& Hickman, J. Measurement of contractile stress generated by cultured rat muscle on silicon cantilevers for toxin detection and muscle performance enhancement. PLOS ONE. 5, doi:10.1371/journal.pone.0011042 (2010).

14. Binnig, G., Quate, C. F., \& Gerber, C. Atomic Force Microscope. Physical Review Letters. 56, 930-933 (1986).

15. Pirozzi, K. L., Long, C. J., McAleer, C. W., Smith, A. S., \& Hickman, J. J. Correlation of embryonic skeletal muscle myotube physical characteristics with contractile force generation on an atomic force microscope-based bio-microelectromechanical systems device. Applied physics letters. 103, 83108, doi:10.1063/1.4817939 (2013).

16. Smith, A., Long, C., Pirozzi, K., \& Hickman, J. A functional system for high-content screening of neuromuscular junctions in vitro. Technology. 1, 37-48, doi:10.1142/S2339547813500015 (2013).

17. Stenger, D. A. et al. Coplanar molecular assemblies of amino- and perfluorinated alkylsilanes: characterization and geometric definition of mammalian cell adhesion and growth. Journal of the American Chemical Society. 114, 8435-8442, doi:10.1021/ja00048a013 (1992).

18. Das, M., Rumsey, J. W., Bhargava, N., Stancescu, M., \& Hickman, J. J. A defined long-term in vitro tissue engineered model of neuromuscular junctions. Biomaterials. 31, 4880-4888, doi:10.1016/j.biomaterials.2010.02.055 (2010).

19. Rumsey, J. W., Das, M., Bhalkikar, A., Stancescu, M., \& Hickman, J. J. Tissue engineering the mechanosensory circuit of the stretch reflex arc: Sensory neuron innervation of intrafusal muscle fibers. Biomaterials. 31, 8218-8227, doi:10.1016/j.biomaterials.2010.07.027 (2010).

20. Das, M., Rumsey, J. W., Bhargava, N., Stancescu, M., \& Hickman, J. J. Skeletal muscle tissue engineering: A maturation model promoting long-term survival of myotubes, structural development of the excitation-contraction coupling apparatus and neonatal myosin heavy chain expression. Biomaterials. 30, 5392-5402 (2009).

21. Das, M. et al. Developing a novel serum-free cell culture model of skeletal muscle differentiation by systematically studying the role of different growth factors in myotube formation. In Vitro Cell Dev Biol Anim. 45, 378-387, doi:10.1007/s11626-009-9192-7 (2009).

22. Stenger, D. A., Pike, C. J., Hickman, J. J., \& Cotman, C. W. Surface determinants of neuronal survival and growth on self-assembled monolayers in culture. Brain research. 630, 136-147 (1993).

23. Hickman, J. J. et al. Rational pattern design for in vitro cellular networks using surface photochemistry. Journal of Vacuum Scienc., \& Technology A: Vacuum, Surfaces, and Films. 12, 607-616 (1994).

24. Das, M., Molnar, P., Devaraj, H., Poeta, M., \& Hickman, J. J. Electrophysiological and morphological characterization of rat embryonic motor neurons in a defined system. Biotechnology progress. 19, 1756-1761, doi:10.1021/bp034076l (2003).

25. Rumsey, J. W. et al. Node of Ranvier formation on motor neurons in vitro. Biomaterials. 30, 3567-3572, doi:10.1016/ j.biomaterials.2009.03.023 (2009).

26. Murugan, R., Molnar, P., Rao, K. P., \& Hickman, J. J. Biomaterial Surface patterning of self assembled monolayers for controlling neuronal cell behavior. International journal of biomedical engineering and technology. 2, 104-134 (2009).

27. Guo, X., Johe, K., Molnar, P., Davis, H., \& Hickman, J. Characterization of a human fetal spinal cord stem cell line, NSI-566RSC, and its induction to functional motoneurons. Journal of Tissue Engineering and Regenerative Medicine. 4, 181-193, doi:10.1002/term.223 (2010).

28. Varghese, K. et al. A new target for amyloid beta toxicity validated by standard and high-throughput electrophysiology. PLoS ONE. 5, e8643, doi:10.1371/journal.pone.0008643 (2010).

29. Natarajan, A. et al. Patterned cardiomyocytes on microelectrode arrays as a functional, high information content drug screening platform. Biomaterials. 32, 4267-4274, doi:10.1016/j.biomaterials.2010.12.022 (2011).

30. Davis, H. et al. Rat Cortical Oligodendrocyte-Embryonic Motoneuron Co-Culture: An Axon-Oligodendrocyte Interaction Model. Journal of biomaterials and tissue engineering. 2, 206-214 (2012).

31. Natarajan, A., DeMarse, T., Molnar, P., \& Hickman, J. Engineered In Vitro Feed-Forward Networks. J Biotechnol Biomater. 3, 2 (2013). 